\title{
"Analysis of Sensitivity of Optical Mach Zehnder Interferometer (OMZI) Sensor Platform using Rib Waveguides"
}

\author{
P. B. Patel ${ }^{*}$, S. T. Hamde ${ }^{2}$ \\ Dr. D. Y. Patil Institute of Technology, Pimpri, Pune-18, Maharashtra, India ${ }^{1}$ \\ S.G.G.S. Institute of Engineering and Technology, Nanded, Maharashtra, India ${ }^{2}$
}

\begin{abstract}
The proposed analysis describes an Optical Mach Zehnder Interferometer (OMZI) sensor platform using rib waveguides. OMZI is a device that makes accurate measurements of Refractive Index (RI), deals with interferometer using single mode transmission. Using the OMZI waveguide structure, optical technique for sensing and measurement was demonstrated. The entire Mach Zehnder Interferometer structure has been made using rib waveguide. Technical considerations for analysis of single mode rib waveguides and its effects on sensitivity are reported. The rib height and width for single mode rib waveguide greatly affects light guiding. Waveguide structure is designed using RSOFT CAD tool. Its optimization and measurement of refractive index has been carried out using Beam Propagation Method (BPM) technique. This is cost effective and simple methodology which can be used in guided wave bio-sensors application.
\end{abstract}

Keywords: Interferometer, Mode, Rib Waveguide.

\section{INTRODUCTION}

Mach-Zehnder Interferometer (MZI) has been widely used by researchers for developing various devices considering its advantage of simple design allowing its easy implementation for different applications ${ }^{[1]}$. MZI has been implemented in switching and modulating purposes, optical interconnect applications, telecommunications, chemical sensing and biosensor applications etc ${ }^{[2,3]}$.Silicon and polymer are used as MZI material. Polymers are more flexible, compatible and cost effective as compared to silicon material ${ }^{[4,5]}$. Hence, combing the properties of polymer materials with the advantages of an MZI used in a multiple sensor technology to develop a biological sensor and sensor array with a simplified micro fabrication technology will yield a very effective, efficient, simple and a useful waveguide device ${ }^{[6]}$. The Optical Mach Zehnder Interferometer (OMZI) is an optical version of the classical Mach Zehnder Interferometer. They provide a basic structure in integrated optics with a chemo-optical interface layer.The proposed rib waveguide structure provides easy integration with optical fiber system. It has potential to realize remote measurement and also in the area of Internet of Things (IoT). It has great advantages of miniaturization and easy integration as compared to conventional measurement technique. The structure of paper is as follows: The rib waveguide structure is explained in the section 2. The modes are analyzed and optimization of rib waveguide for OMZI is highlighted in section 3. In section 4, sensitivity of complete OMZI structure is analyzed with its application in measurement of refractive index of the sample. Conclusions are drawn in section 5.

\section{STRUCTURE OF RIB WAVEGUIDE}

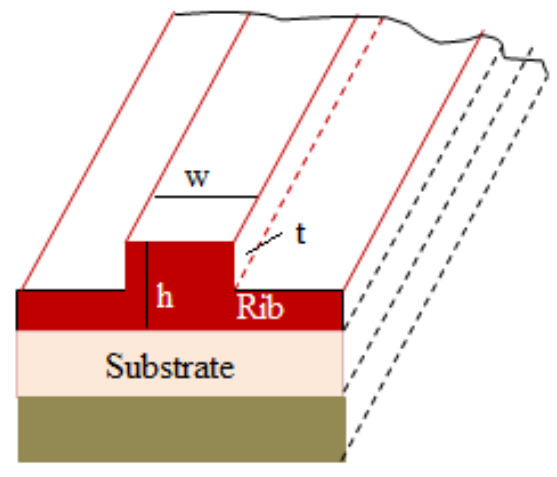

Fig 1. Structure of rib waveguide 


\title{
International Journal of Innovative Research in Electrical, Electronics, Instrumentation and Control Engineering
}

\author{
Vol. 6, Issue 12, December 2018
}

A homo waveguide structure and hetero waveguide structure are basic elements of integrated optics systems. In this work, a rib waveguide is used for OMZI analysis which is a multilayer geometry ${ }^{[7]}$.

It is characterized by a substrate, a slab, rib, and a cover. The substrate surface begins at $y=0$, and is a single index that extends semi-infinitely in the $\mathrm{y}$ direction. The slab extends from $\mathrm{y}=0$ to $\mathrm{y}=\mathrm{Slab}$ Height, and is treated as infinite in $\mathrm{x}$ and $\mathrm{z}$. Each ridge is represented by a waveguide segment, and obeys the Component ( $\mathrm{y}=0$ to $\mathrm{y}=$ slab height) and Component width parameters. Figure 1 shows the rib waveguide structure under consideration for the proposed structure. Single mode propagation of light is main design requirement for the rib wave guide ${ }^{[8]}$.

\section{NUMERICAL ANALYSIS}

The main requirement is to ensure a mono-mode propagation of light through the rib waveguide. This depends on specific dimensions (height and width) of waveguide under consideration ${ }^{[9]}$.

Figure 2 shows the refractive index profile for proposed rib waveguide structure.

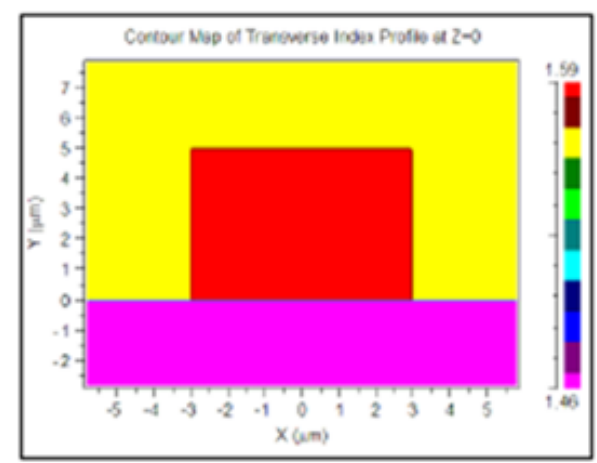

Fig. 2 Index profile of rib waveguide at $z=0$

The analysis of wave equation given in equation 1 explains the information about light travelling through the waveguide. (Core mode and cladding mode) ${ }^{[10]}$. A conventional optical fiber supports light modes as it is a weakly coupled waveguide. The input light modes along the rib waveguide are Linearly Polarized (LP) modes ${ }^{[11]}$. Using scalar wave equations, we can calculate propagation constants for LP modes.

Figure 3 (a) \& (b) shows mode calculation with change in rib width. The electric field propagation in the $z$-axis into OMZI satisfies scalar wave equation. It is given by equation $1^{[12]}$.

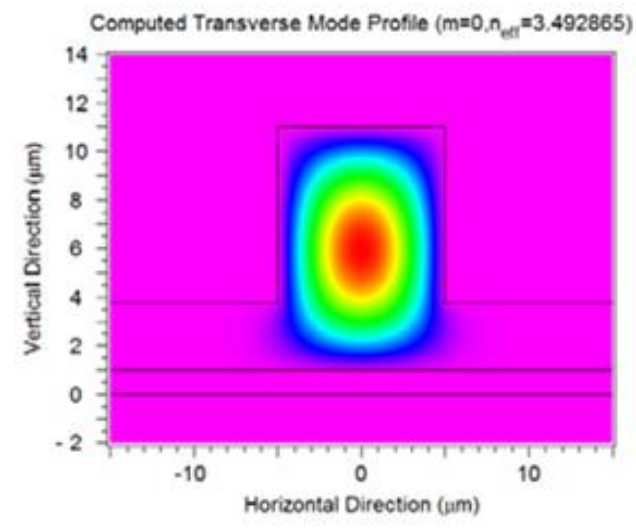

Fig 3(a) Mode calculation with $10 \mu \mathrm{m}$ rib width

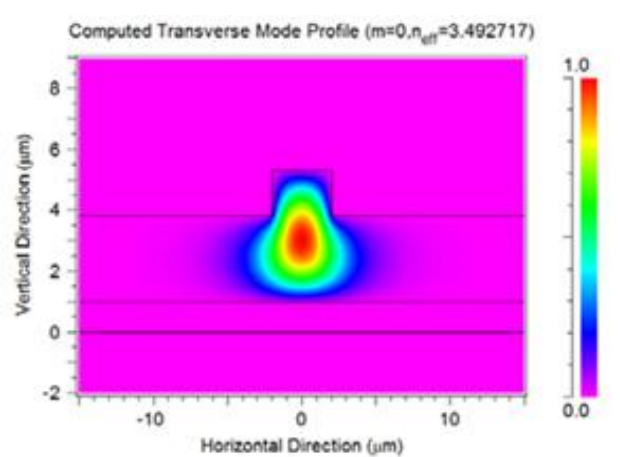

Fig. 3 (b) Mode calculation with $5 \mu \mathrm{m}$ rib width

$$
\left(\frac{\partial^{2}}{\partial r^{2}}+\frac{1 \partial}{r \partial r}+\frac{1 \partial^{2}}{r^{2} \partial \emptyset^{2}}+k^{2} n^{2}-\beta^{2}\right) \varphi=0 \quad \text {...eq. (1) }
$$


Vol. 6, Issue 12, December 2018

where,

$\varphi=$ Electric field,

$k=$ Free space wave number,

$n=$ Refractive index and

$\beta$ is the propagation constant ${ }^{[13,14]}$.

3a. Proposed OMZI Structure

The proposed sensor platform using OMZI configuration essentially has Y- splitter and Y-coupler branch. Both of these $\mathrm{Y}$ branches of interferometer structures are designed by considering low losses as well as no cross coupling between measurement arm and reference arm. Figure 4 shows proposed OMZI structure. The external physical parameter at measuring arm is responsible for change in phase shift of light at measuring arm. This variation is recorded at out monitor ${ }^{[2]}$.

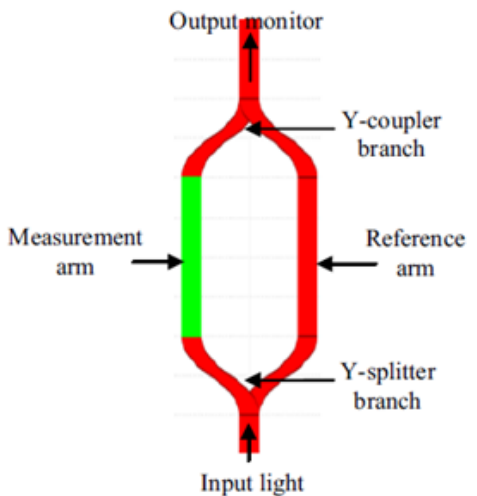

Fig. 4 Proposed OMZI structure

The rib width, rib height and Y- branch length are variable parameter. The detection sensitivity of overall structure to change in refractive index is dependent on the waveguide dimension. Sensitivity is defined as the ratio of change of effective refractive index of guided mode to the rate of refractive index of cover media. The output and input power of the OMZI is given as follow,

$\frac{\text { Pout }}{\text { Pin }}=\frac{1}{2}[1+\cos \emptyset]$

$\emptyset=k_{0} L\left(n_{\text {eff, sensor }}-n_{\text {eff, ref }}\right) \quad \ldots$ eq. (3)

$k_{0}=2 \pi / \lambda$, a wave number (free space),

$\mathrm{L}$ is sensing length

$\left(n_{e f f, \text { sensor }}-n_{\text {eff,ref }}\right)$, index difference between measurement and reference path

Surface sensitivity $\mathrm{S}$ is given as follow,

$S=\frac{\partial n_{\text {eff }, \text { sensor }}}{\partial \eta}$

Change of phase shift and sensitivity is related as ,

$\emptyset=k_{0} L S \Delta \eta$ ...eq $(5)$

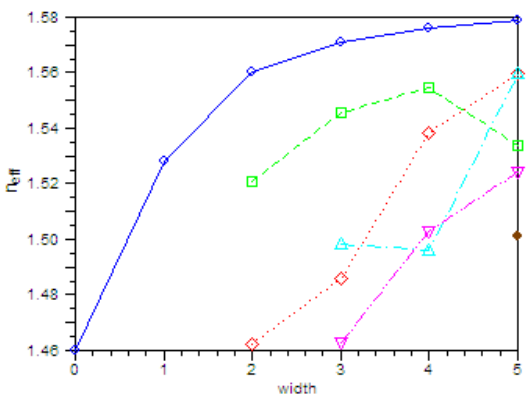

Fig. 5 Effects of rib width on mode propogation 


\section{International Journal of Innovative Research in Electrical, Electronics, Instrumentation and Control Engineering}

Vol. 6, Issue 12, December 2018

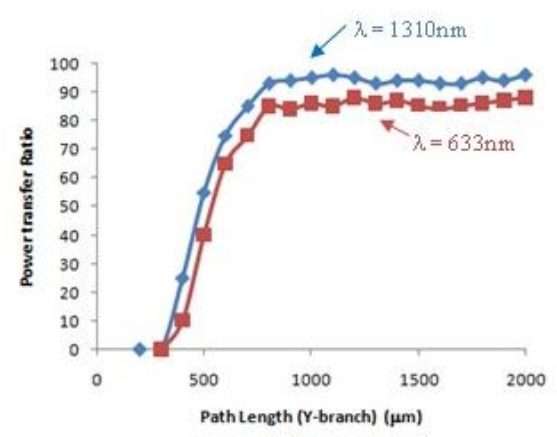

Fig.6 Power transfer in $\mathrm{Y}$ branch

\section{RESULTS AND DISCUSSION}

The OMZI is designed to know the behavior of the device and determine its physical parameters for analysis. An OMZI with a core less than 10 microns (height and width) are designed using the RSOFT CAD tool and is tested in simulation software called Beam PROP. Figure 5 highlight the effect of rib width on mode propagation for the proposed structure, to maintain mono mode propagation, width of rib is important. Figure 6 shows numerical analysis of power transfer in Y-branch as a function of the length variation at wavelength $1310 \mathrm{~nm}$ and $633 \mathrm{~nm}$. The designed OMZI being a waveguide, for which, the integration area depends upon the type of waveguide structure; the program will integrate over a rectangle with width and height determined by the waveguide parameters.
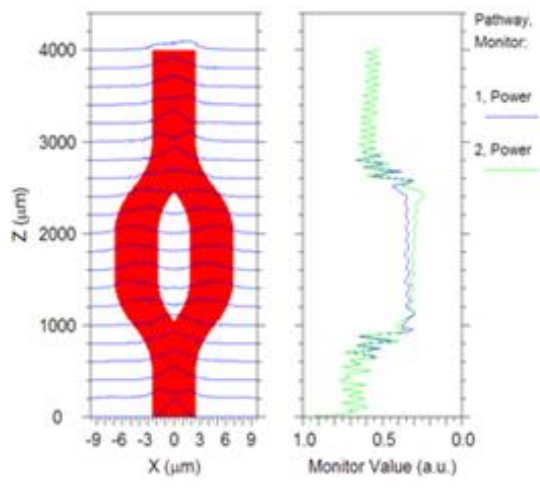

Fig.7 Rib waveguide OMZI

The waveguide output monitors selection depends on the structure designed for OMZI. The waveguide analysis done at various wavelengths proved that rib waveguide has good wave-guiding properties at all three wavelengths. Here the interacting area is more as compared to other waveguide structure (like channel waveguide). The complete designed OMZI using rib waveguide is shown in figure 7 is used for the detection of Refractive Index (RI) of sample. The results shown in figure 8 confirmed changes in the refractive index with respect tothe changes in wavelength for the rib waveguide under consideration. For optimization of rib waveguide for low propagation losses, different rib width (w) and height (h) are tested and analysed to find its effects on light intensity into the waveguide. Figure 9 shows effects of variation in rib waveguide width and height on intensity of evanescence field.

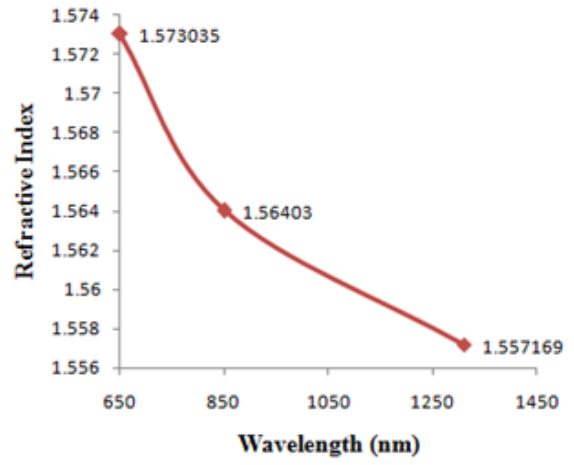

Fig. 8 Refractives indices of waveguide for vrious wavelength 


\section{International Journal of Innovative Research in Electrical, Electronics, Instrumentation and Control Engineering}

Vol. 6, Issue 12, December 2018

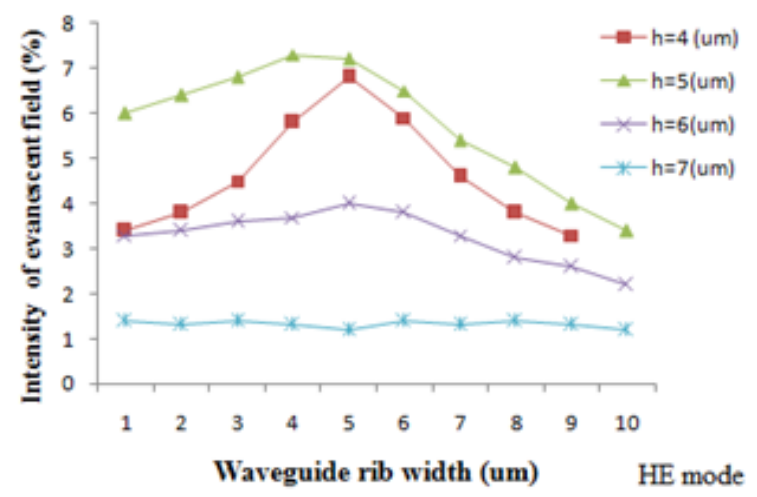

Fig. 9 Intensity variation with respect to rib width

As shown in figure 4 and 7, the measurement arm is the interacting area where the refractive index change takes place with respect to change in physical parameter at measurement arm. The upper layer of this waveguide structure is analogous to the optical cladding layer made up of polymer having lower refractive index than that of the waveguide core. This layer absorbs chemical from the surrounding area. The refractive index of polymer changes as the concentration of the absorbed sample chemical changes.

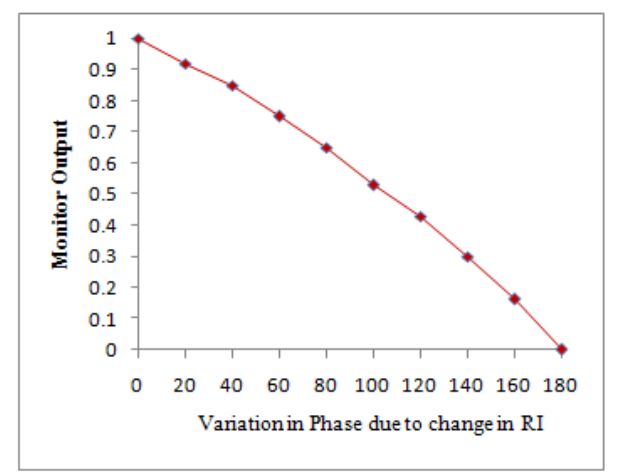

Fig. 10 Monitor output variation with change in phase due to change in RI

This causes a phase shift in the measurement arm with respect to the reference arm. The waveguide structure is designed to operate with relatively stronger evanescent wave coupling between the outer polymer layer and the light propagating along the waveguide core. With this interaction, chemically induced changes in the refractive index of the polymer causes considerable changes in the phase shift. The changes in the phase shift equally produces changes in the monitor output of the waveguide sensor platform. The interferometer based on optical waveguide mainly works on evanescent field penetration and phase shift measurement. Near surface region of a sensor area with the evanescent field, any change of the refractive index of the sample volume induces a phase shift of the guided mode compared to a reference field typically of a mode propagating through the reference arm of the same waveguide structure. This is shown in figure 10 .

\section{CONCLUSION}

The polymer waveguide sensor platform and its sensing property is analysed. The performance of the rib waveguide structure is limited by the level of light scattering out of the plane due to the side wall layer roughness, light penetration depth and evanescence field informality. In order to meet the requirement of practical sensing and interrogation application, the rib waveguide parameters can be controlled easily. According to the analysis, the sensitivity depends on the rib thickness and rib width. From the results obtained, it is observed that waveguides with rib width below $4 \mu \mathrm{m}$ and height $5 \mu \mathrm{m}$ shows mono mode behavior. So, we can conclude that OMZI can be used for sensing applications. Rib waveguides are analysed for the wavelength variation from $\lambda=630$ up to $1310 \mathrm{~nm}$. This structure can be tuned for specific application. The OMZI analysis is used for measurement of refractive index of sample. The current advances in technology will help further in reduction of the losses in the rib waveguides. This will enable the OMZI based structure for various bio-sensing applications. 


\section{International Journal of Innovative Research in Electrical, Electronics, Instrumentation and Control Engineering}

Vol. 6, Issue 12, December 2018

\section{ACKNOWLEDGEMENT}

This work was part of the RPS scheme of Savitribai Phule Pune University, (SPPU), Pune, INDIA.

\section{REFERENCES}

[1]. Dengpeng Yuan, Y. Dong, Y. Liuand T. Li, "Mach-Zehnder Interferometer Biochemical Sensor Based on Silicon-on-Insulator Rib Waveguide with Large Cross Section", Sensors, 15, 21500-21517, (2015).

[2]. P. B. Patel and S. T. Hamde, "Emerging Waveguide Technology" (edited by Kok Yeow You, University of Malaysia), Intech Publication, Chapter 5, 81-92, (2018).

[3]. P. B. Patel, S. T. Hamde, "Analysis of the Mach-Zehnder Interferometer Waveguide Structure for Refractive Index easurement" Journal of Optics, Springer Publication, 46 (4), 398-402, (2017).

[4]. F. Dullo, J. Tinguely, S. Solb, and O.Helles, "Single mode limit and bending losses for shallow rib Si3N4 waveguides," IEEE Photon Jolurnal, 7, 1-11, (2015).

[5]. P. Kozma, F. Kehl, E. Ehrentreich Förster, C. Stamm, and F. Bier, "Integrated planar optical waveguide interferometer Biosensors: a comparative review," Biosensor Bioelectron. 58, 287-307 (2014).

[6]. A. Densmore, D. X. Xu, S. Janz, P. Waldron, J. Lapointe, T. Mischki, G. Lopinski, "Sensitive label-free bio-molecular detection using thin silicon waveguides," Advanced Optical Technology, 725967 (2008).

[7]. A. Siarkowski, L. Hernandez, B. Borges, and N. Morimoto, "Sensing based on Mach-Zehnder Interferometer and Hydrophobic Thin Films used on Volatile Organic Compounds Detection,"Optical Eng.,51, 054401 (2012).

[8]. Soref, R. A., Sshmidtchen, J., Petermann, K., "Large single-mode rib waveguides in GeSi-Si and Si-on-SiO2", IEEE Journal of Quantum Electronics, Vol. 27, (8), 1971-1974, (1991).

[9]. Fischbeck, G., Moosburger, R., Topper M.,Petermann, K., “Design concept for single mode polymer waveguides”, Electronics Letters, vol. 32, (3), 212-213, (1996).

[10]. D. Esinenco, S. Psoma, M. Kusko, A. Schneider, and R. Muller, "Su-8 micro-biosensor based on mach-zehnder interferometer", Rev. Adv. Mater. Sci, 10 (4), 295-299, (2005)

[11]. R. Bruck and R. Hainberger, "Polymer waveguide based biosensor", Photonics,Devices, and Systems Iv, pages 71380. International Society for Optics and Photonics, (2008).

[12]. A. Crunteanu, M. Pollnau, G. Janchen, C. Hibert, P. Hoffmann, R. Salath'e, R. Eason, C. Grivas, and D. Shepherd, “ Ti: sapphire rib channel waveguide fabricated by reactive ion etching of a planar waveguide", Applied Physics B: Lasers and Optics, 75(1):15-17, (2002).

[13]. A. Rickman, G. Reed, and F. Namavar, "Silicon-on-insulator optical rib waveguide loss and mode characteristics", Lightwave Technology, Journal of, 12(10), 1771-1776, (1994).

[14]. B. Sepulveda, J. S. Del Rio, M. Moreno, F. Blanco, K. Mayora, C. Dominguez, and L. Lechuga, "Optical biosensor microsystems based on the integration of highly sensitive Mach-Zehnder interferometer devices", Journal of optics A: pure and applied optics, 8 (7), S561, (2006)

\section{BIOGRAPHIES}

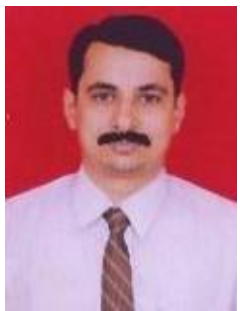

Mr. P. B. Patel is working as Associate Professor in Instrumentation department at Dr. D. Y. Patil Institute of Technology (DIT), Pune. He has completed B.E. (Instrumentation) from North Maharashtra University (NMU), Jalgaon and M.E. (Instrumentation) from Government College of Engineering, Pune University. He has also completed PGDMM (Marketing Management) from Indian Institute of Cost and Management Studies and Research, Pune University. He has secured first rank during PGDMM course at the Institute and second rank at Pune University. Presently he is doing his Ph.D. at SGGSIET, Nanded. His research area is interferometer based fiber optics sensors. He has over 22 years of experience in Industry, teaching, academic planning, administration, training and campus recruitments etc. to his credit with several top Institutions such as Pune Institute of Computer Technology (PICT), Pune and DIT, Pimpri, Pune amongst others affiliated to Pune University, Maharashtra. He is Fellow-IEI (FIE) and Life Member ISTE, BMESI, ISOI, ISA and IETE. He has published 30 papers at National / International conferences and 05 papers at technical Journals. He is reviewer for "Optical Fiber Technology" Elsevier publication Journal. He has also published one chapter for Intech Publisher on Waveguide Technologies.

He has worked as Executive Committee member for IETE and IEI, Pune center. He has completed international certification from "DALE CARNEGIE" in "High Impact Teaching Skills" under Mission 10X program of Wipro Technologies. He has signed MoU with various organizations like, Infosys Ltd., B and R automation, Schneider, Yamaha Motors, Sungard etc. He has an impressive record in mentoring students and academic leadership.

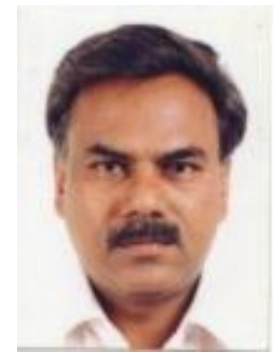

Dr. S. T. Hamde is working as Professor and Head of Department of Instrumentation Engineering Sant Guru Gobind Singji Institute of Engineering and Technology (SGGSIET), Nanded. He has completed B.E and ME from Dr. B. A. Marathwada University Aurangabad and Ph.D from IIT, Roorkee.

He has carried research work in the field of Biomedical Engineering, particularly on bioelectric signals. Effective use of wavelet transform in ECG signal analysis and interpretation is proved to be of immense help to society. Use of wavelet transforms for heart rate variability (HRV), ventricular late potential (VLP) and His potential and in ECG data compression has been carried out. Work is continued to develop reliable automated cardiac disease diagnosis system. He had 


\title{
International Journal of Innovative Research in Electrical, Electronics, Instrumentation and Control Engineering
}

\author{
Vol. 6, Issue 12, December 2018
}

guided 10 Ph.D. students and 06 students are doing Ph.D. under his guidance. Ha has worked as a editorial board member for National and International conference proceedings. He has received Khosla Annual Research Award 2001.

(The research paper titled ECG Data Compression using Non-redundant emplates has got the First Khosla Research Prize Rs.3000/ and a MEDAL of I.I.T. Roorkee.) He did training on ECG signal processing at CORE laboratory of University of Glasgow, Scotland, UK during June, 2006 jointly working on a project to establish ECG database from healthy native population. Coordinators are Professor P. W. Macfarla le, who is a Director of Clinical Research center, University of Glasgow, Scotland, Prof Vinod Kumar from IIT, Roorkee and Dr. Dilbag Singh from NIT, Jalandhar. 